\title{
Ultrasound assessment of great saphenous vein insufficiency
}

\section{Rajiv K Chander' \\ Thomas S Monahan ${ }^{1,2}$ \\ 'Section of Vascular Surgery, Department of Surgery, University of Maryland School of Medicine, ${ }^{2}$ Department of Surgery, Baltimore Veterans Affairs Medical Center, Baltimore, MD, USA}

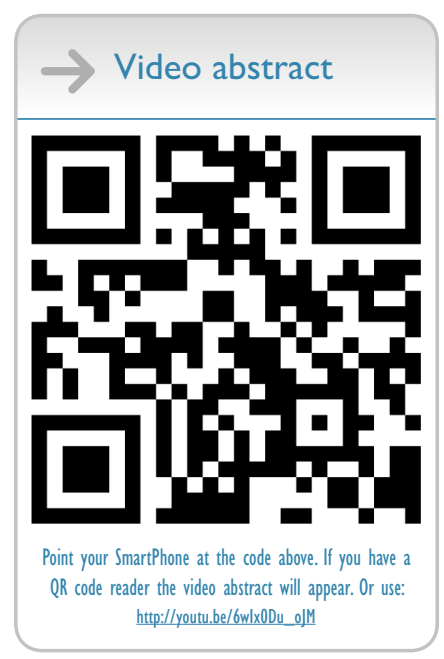

Correspondence: Thomas S Monahan Section of Vascular Surgery, Department of Surgery, University of Maryland School of Medicine, 22 South Greene Street, Baltimore, MD 2I 20I, USA

Tel + I 4103285840

Fax +I 4103280717

Email tmonahan@smail.umaryland.edu
This article was published in the following Dove Press journal:

Journal of Vascular Diagnostics

15 June 2015

Number of times this article has been viewed

\begin{abstract}
Duplex ultrasonography is the ideal modality to assess great saphenous vein insufficiency. Duplex ultrasonography incorporates both gray scale images to delineate anatomy and color-Doppler imaging that visualizes the flow of blood in a structure. Assessment of great saphenous vein requires definition of the anatomy, augmentation of flow, evaluation for both superficial and deep vein thrombosis, and determining the presence of reflux. Currently, evolution in the treatment of reflux also relies on ultrasound for the treatment of the disease. Understanding the utilization of the ultrasound for the diagnosis and treatment of greater saphenous vein reflux is important for practitioners treating reflux disease.
\end{abstract}

Keywords: duplex ultrasonography, small saphenous vein

\section{Introduction}

Venous insufficiency is a highly prevalent, but often unrecognized, substantial source of morbidity in Western society. Venous insufficiency is the inability for veins to transport blood from the lower extremities back to the heart. Venous insufficiency can arise from obstruction, such as in the case of deep vein thrombosis (DVT) or valvular incompetence as in saphenous reflux or postthrombotic syndrome. One sequelae of venous insufficiency is varicose veins. Estimates of the prevalence of varicose veins range from $2 \%$ to $56 \%$ in men and $<1 \%$ to $73 \%$ in women. The wide variation in prevalence could be explained by multiple factors including different practices for screening and diagnosis as well as inherent differences in patient populations. ${ }^{1}$ Each year in the United States over 20,000 patients are diagnosed with new venous ulcers. ${ }^{2}$ The cost for the care of patients with chronic venous disease is estimated to be between $\$ 150$ million and $\$ 1$ billion each year. ${ }^{3}$

Duplex ultrasound allows simultaneous evaluation of both anatomy and physiology. Ultrasound is essential in all phases of management of patients with chronic venous insufficiency - preoperative evaluation, intraoperative performance catheter-based procedures, and postoperative evaluation. Furthermore, duplex ultrasound is costeffective and safe for the patient. Components essential to the evaluation of chronic venous insufficiency with ultrasound are visualization of the anatomy, compressibility, provocative maneuvers to assess reflux, and augmentation of flow. ${ }^{4}$

\section{Diagnosis}

An understanding of lower extremity venous anatomy and its common variations is essential for the performance of duplex ultrasound, interpretation of the images, decision 
for surgery, and ultimately, the execution of radiofrequency ablation. In recent years, the nomenclature of the lower extremity venous system has been modified to eliminate confusion. The principle veins of the deep system are the tibial veins, which form a confluence and become the popliteal vein. The popliteal vein is adjacent to the popliteal artery and becomes the femoral vein as it crosses Hunter's canal in the adductor magnus muscle. The femoral vein was formerly referred to as the superficial femoral vein because it lies adjacent to the superficial femoral artery. This terminology has been changed to eliminate confusion; the femoral vein is a deep vein. The principle veins of the superficial system are the great saphenous vein (GSV), formerly known as either the greater or long saphenous vein, and the small saphenous vein (SSV), formerly known as the short, smaller, or lesser saphenous vein. ${ }^{5}$ The GSV arises from the veins of the foot and courses anterior to the medial malleolus and along the medial aspect of the lower leg and along the medial aspect of the thigh. The GSV joins with the common femoral vein (CFV) of the deep system at the saphenofemoral junction (SFJ) in the groin. The GSV is located between the superficial fascia and the deep fascia of the leg. Its location between these fascial layers gives the GSV the appearance of "Cleopatra's Eye" (Figure 1). The SSV originates on the posterior leg and is likewise located between the superficial and deep fascia of the leg. It is located between the two heads of the gastrocnemius muscle and enters the popliteal vein at the saphenopopliteal junction.

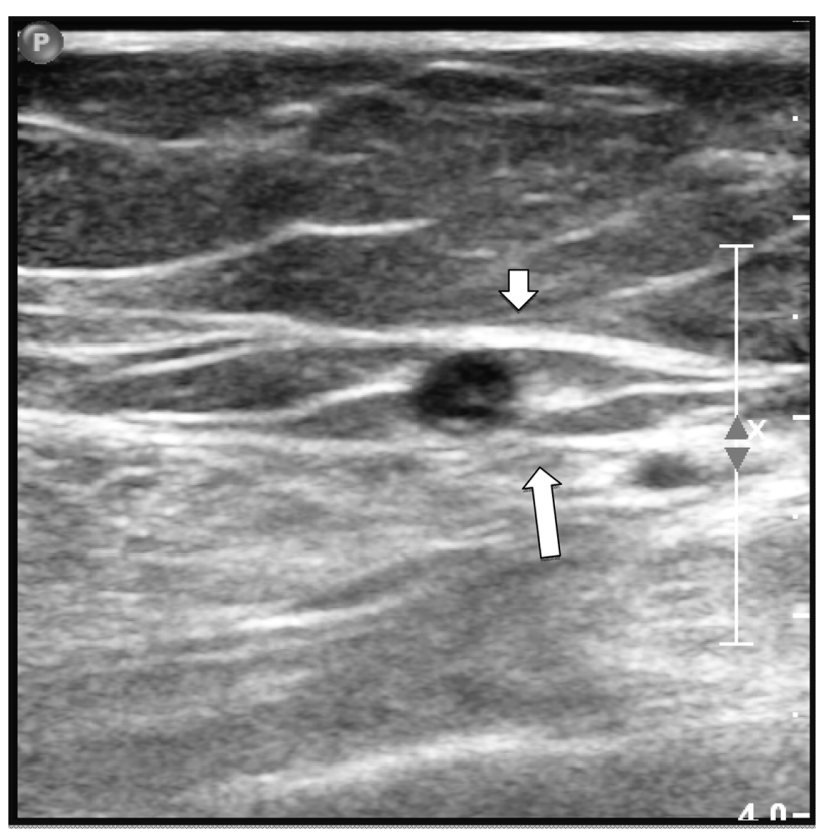

Figure I Gray scale image of the great saphenous vein (GSV) in the thigh. Notes: The GSV is identified by its location between the superficial (short arrow) and deep (long arrow) fascia. This appearance has been referred to as "Cleopatra's Eye."
Venous anatomy has more variation than arterial anatomy. When treating symptomatic venous disease, it is important to understand the common variants of venous anatomy. There are two clinically relevant, common anatomic variants, the intersaphenous vein and the anterior accessory saphenous vein. The intersaphenous vein, formerly known as the vein of Giacomini, is a communicating branch that connects the SSV to the GSV. The anterior accessory saphenous vein runs along the anterior surface of the thigh. Like the GSV, it lies between a superficial fascial layer and the deep fascia on the anterior surface of the thigh muscles. It joins the deep system at the SFJ.

The superficial system communicates with the deep system at multiple points in the leg and thigh. It is estimated that these veins can number up to 150 and are of varying location, length, and importance. These were formerly known by their eponyms: the perforating vein of Dodd, Boyd, and Cockett. In the present nomenclature, they are named by their anatomic locations. There are four groups of perforating veins that are more consistently present and are of clinical significance when evaluating patients with venous insufficiency. These are the inguinal perforators connecting the GSV with the femoral vein in the thigh, the perforators of the femoral canal connecting the GSV to the femoral vein in the distal thigh (Figure 2), the paratibial perforators connecting the GSV with the posterior tibial veins in the proximal lower leg and the posterior tibial perforators connecting the GSV with the posterior tibial veins in the lower medial aspect of the lower leg. ${ }^{6}$ These veins when incompetent can transmit the high pressures of the deep system to the superficial system causing

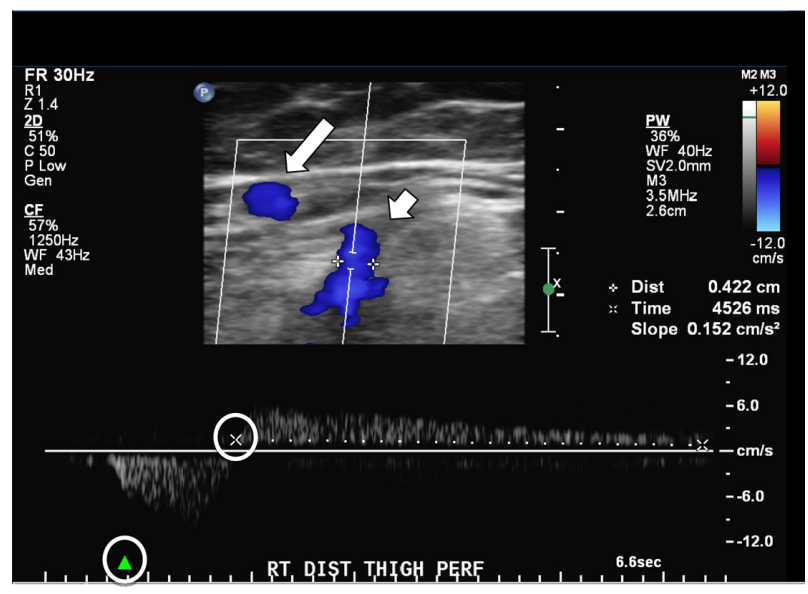

Figure 2 Perforating vein with reflux.

Notes: Color-Doppler is used to identify the great saphenous vein (GSV) in its fascial sheath (long arrow). A $4.2 \mathrm{~mm}$ perforating vein (short arrow) communicates with the GSV. Reflux is determined by compressing and rapidly releasing the distal leg (green arrow) and duration of reversal of flow is identified and measured (x) at the beginning of flow reversal. In this case, flow is reversed for 4.5 seconds. 
or exacerbating varicosities of the superficial system. Incompetence of the perforating veins allows direct transmission of the elevated pressures generated by the calf muscle pump to the superficial system. Perforating veins larger than $3.5 \mathrm{~mm}$ should be noted and considered significant during the ultrasound evaluation for chronic venous insufficiency. ${ }^{4}$

The second component of the ultrasound evaluation for venous insufficiency is assessment of compressibility. Compressibility is assessed in both the deep and superficial systems. Compressibility is an accurate and reliable assessment for venous thrombus. A normal vein should be completely compressible when imaged in the transverse plane (Figure 3). Acute thrombus is manifested by an enlarged, noncompressible vein, whereas veins with chronic thrombus tend to be smaller in caliber and have echogenic

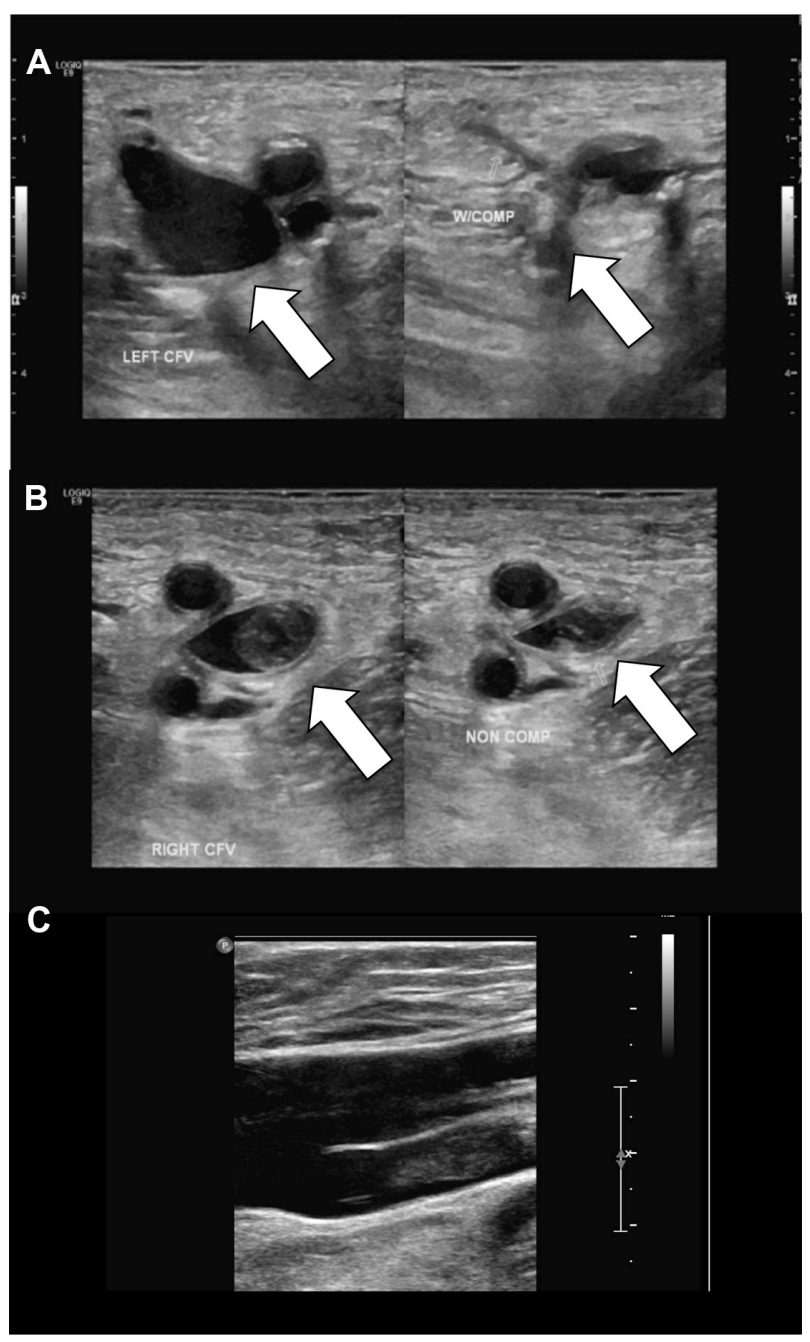

Figure 3 Gray scale images of deep vein thrombus.

Notes: The normal left common femoral vein (CFV, arrows) is visualized at the saphenofemoral junction. The vein can be completely compressed (W/COMP, panel A). When deep vein thrombus is present the CFV can not be compressed (NON COMP, panel B). Thrombus is seen in the right. CFV cannot be compressed due to the presence of thrombus. Transverse images demonstrate the thrombus extending to the (panel $\mathbf{C}$ ). walls reflective of the fibrous changes that occur with the maturation of thrombus. ${ }^{7}$ Presently, the only absolute contraindication for radiofrequency ablation of the GSV is thrombus in the segment of vein to be ablated. This contraindication reflects the risk of pulmonary embolism associated with passing the catheter past the thrombus.

The third component of the ultrasound evaluation for venous insufficiency is the assessment of reflux. Ultrasonography is the standard for assessing venous reflux in GSV and SSV as well as accessory saphenous veins: 41\% of people have anterolateral saphenous vein, $95 \%$ of people have a posterior thigh extension of the $\mathrm{SSV}^{8}$ and $70.4 \%$ of people have an intersaphenous vein. ${ }^{9}$ The superficial veins, the deep veins, perforator veins, and all variant branches need to be evaluated with color-Doppler ultrasound for a complete evaluation. ${ }^{9,10}$ Patient positioning is an important consideration for the evaluation of venous reflux. It is apparent that the supine position is optimal to define venous anatomy and assess for compressibility. However, there is little consensus concerning the optimal patient position to assess reflux. Recommendations for patient positioning range from $10^{\circ}$ of reverse-Trendelenburg tilt to having the patient standing erect. Although there is little consensus, venous reflux has been consistently demonstrated in this array of patient positions. ${ }^{11}$ Often, patients with venous insufficiency have difficulty standing for the duration of time required to perform the reflux component of the examination. Additionally, the standing position makes the examination more technically challenging for the sonographer. Our practice is to position the patient in $20^{\circ}$ of reverse-Trendelenburg tilt.

After the patient is positioned, a provocative maneuver is performed to demonstrate the reversal of flow in the vein studied. For veins of the proximal thigh, a Valsalva maneuver is used to evoke reflux. For the more distal veins, the leg distal to the segment of vein being interrogated is rapidly compressed and released. Compression can be accomplished with either a blood pressure cuff, or as in our laboratory, by manual compression. Reflux is manifest by a rapid antegrade flow coincidental with compression followed by retrograde flow upon release of pressure (Figure 4). Pathologic reflux is defined by reversal of flow greater than 500 milliseconds in duration. ${ }^{12}$ Some authors suggest that the threshold for identification of reflux in the deep system should be increased to 1 second in duration. ${ }^{4}$ It is not uncommon for patients with symptomatic GSV insufficiency to have reflux several seconds in duration.

The final component of the ultrasound evaluation for venous insufficiency is assessment of augmentation of flow. 


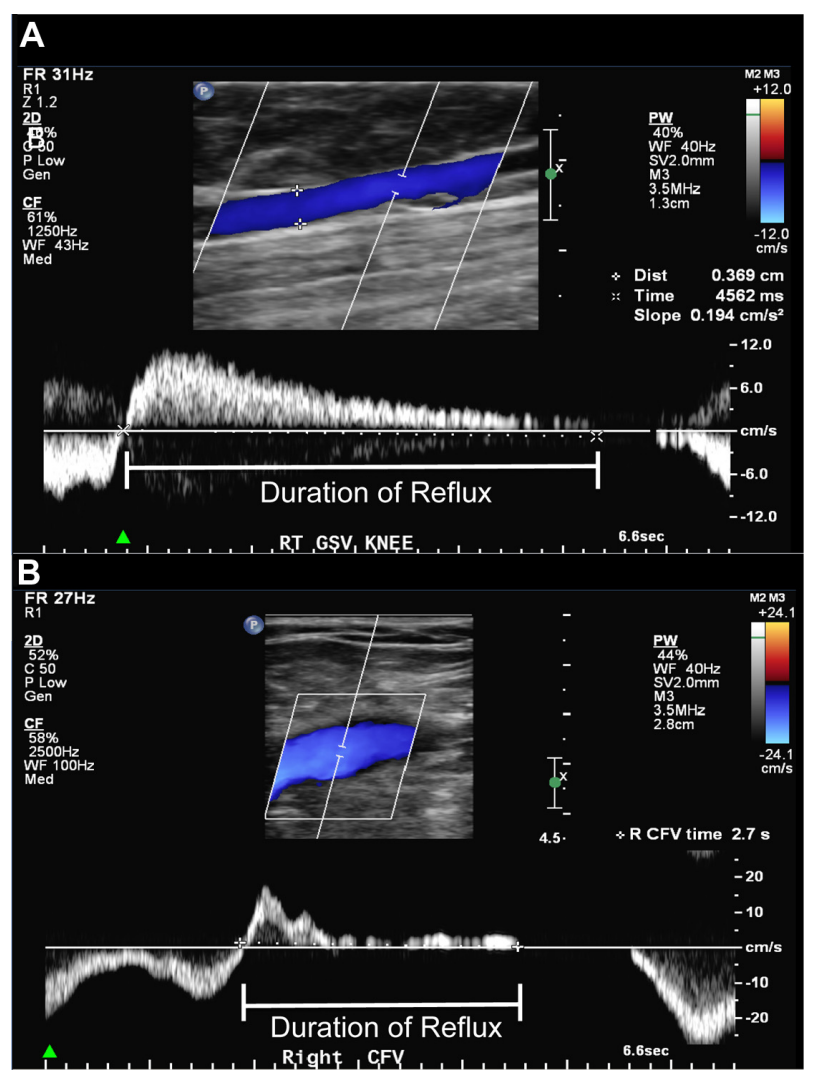

Figure 4 Superficial and deep venous reflux.

Notes: Reflux is measured while imaging the vein in the transverse orientation. The proximal end of the vein is on the left side of the image. In the examination of the great saphenous vein, the vein is imaged in the transverse section with colorDoppler ultrasound. The distal calf is rapidly compressed and released. The duration of flow reversal is measured beginning at the reversal of flow (panel A). Reflux in the deep system is assessed in a similar manner, except the patient is asked to perform a Valsalva maneuver to provoke reflux (panel B).

Augmentation of flow is detected with color-Doppler evaluation for venous insufficiency. Intrathoracic pressure varies throughout the respiratory cycle. In the supine position in a patient with normal anatomy and physiology, intrathoracic pressure is transmitted to the CFV. When intrathoracic pressure exceeds venous pressure, flow in the CFV is momentarily ceased. The result of the cycling intrathoracic pressure is phasicity that occurs at the same rate as the respiratory rate (Figure 5). There is no valve between the right atrium and the vena cava. Thus patients with elevated right heart pressures can transmit the elevated right heart pressure to the veins of the lower extremity. In cases of patients with elevated right heart pressures, such as in heart failure or volume overload, phasicity occurs at a much shorter interval. The popliteal vein studied in Figure 5, panel B, had a phasicity of approximately one cycle per second. This correlated with the observed heart rate of approximately 60 beats/min. Lack of respiratory variation in flow and waveform patterns at rest and during flow augmentation in the CFV suggest proximal obstruction.

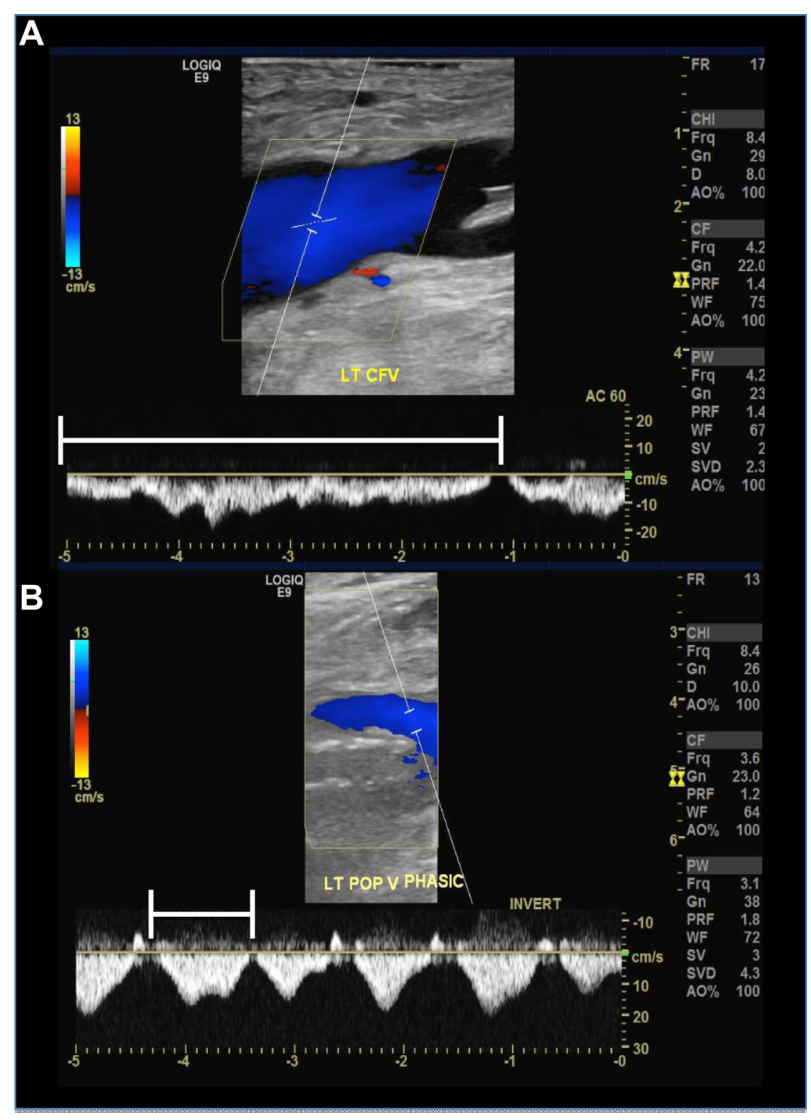

Figure 5 Venous phasicity.

Notes: In an euvolemic patient, normal intrathoracic pressure changes associated with respiration are transmitted to the femoral vein. Panel A demonstrates a 5-second-long period of phasicity, which correlates with the normal respiratory rate of approximately 12 breaths per minute. Patients with elevated right heart pressures, often from volume overload or congestive heart failure, transmit the pressure from the beating right atrium to the veins of the lower extremity. In panel B there is phasicity at approximately one cycle per second correlating with a heart rate of 60 beats per minute. Proximal occlusion results in the absence of phasicity.

\section{Treatment and the role of ultrasonography}

Treatment for varicose veins after failing medical treatment has evolved over the past decade replacing the open surgical surgery with minimally invasive catheter-based therapy performed under local and tumescent anesthesia. Multiple modalities of vein ablation techniques are available and are categorized according to the mode of ablation: mechanical, chemical, or thermal. Mechanical ablation, surgical ligation with or without excision or phlebectomy, has largely been replaced by catheter-based chemical and thermal procedures. Catheter-based techniques have gained immense popularity among both patients and providers. These evolving technologies offer significant advantages such as a lack of an incision, less postoperative pain, less burse, and quicker recovery times. ${ }^{13-21}$ Chemical ablation involves under ultrasound guidance introduction of a catheter into the vessel while under 
vision, introduction of irritant agents into the vein, hence causing endothelial damage and ultimately thrombosis of the treated vessel. ${ }^{22}$ Lastly, thermal ablation involves generation of heat either with radiofrequency energy or laser light at a temperature to denature the proteins that constitute the vein wall resulting in the obliteration of the lumen.

The goals of ablation therapy in patients with symptomatic venous disease are improvement in symptoms as well as appearance. Superficial vein ablation is thought to produce beneficial effects by reducing venous volume in the limb and thereby the effects of venous hypertension upon the cutaneous tissues. ${ }^{23}$

Treatment of chronic venous reflux is managed according to the severity of the disease. With goals to improve symptoms of venous hypertension, the lipodermatosclerosis and heal ulcers are treated. Conservative medical management is recommended initially with leg elevation, exercise, and compression therapy. ${ }^{24,25}$ Persistent symptoms, despite maximal medical management is an indication for surgical intervention. Ablation therapy is thought to produce beneficial effects by reducing venous hypertension and venous volume in the limb and thereby the effects of venous hypertension upon the cutaneous tissues. ${ }^{23}$ Thrombus in the segment of saphenous vein to be ablated is an absolute contraindication to endovenous ablation. Relative contraindications to ablation include pregnancy, acute superficial or deep venous thrombosis, moderate to severe peripheral artery disease, advanced generalized systemic disease and joint disease that interferes with mobility. These patients might be better treated with a GSV ligation or medical therapy (compression and elevation) alone.

Ultrasonography is essential for catheter-based thermal ablation procedures. First, understanding the components of the evaluation previously outlined influences the procedure to be performed. It is important to understand what segments of vessels have reflux. Preoperative evaluation identifies superficial vessels in addition to the GSV that are causing reflux such as the anterior accessory saphenous vein, the intersaphenous vein, and SSV. The preoperative evaluation also informs the practitioner of a large perforator vein that has reflux. Correlating the preoperative findings with the patient's symptoms allows the procedure to be optimized for the individual patient. Additionally, data from the preoperative evaluation will better inform the patient positioning (supine, prone, or frog-legged) and the selection of catheters used in the procedure.

The ultrasound is utilized in all catheter-based procedures. Gray scale ultrasound guidance allows for more efficient canalization of the vessel to be treated. The GSV, especially in younger patients, can be very reactive and go into vasospasm as cannulation is attempted. Ultrasound guidance increases accuracy of cannulation and decreases the number of attempts and thus the incidence of procedure-limiting vasospasm. Cannulation is best performed with the patient in the reverse-Trendelenburg position. After cannulation is achieved, tumescent anesthesia is administered in the periadventitial space under ultrasound guidance. The tumescent anesthesia will provide anesthesia and decreases the heat to the surrounding tissue by absorbing it. In this step of the procedure, it behooves the practitioner to be familiar with the gray scale appearance of the GSV. To be effective, tumescent fluid needs to be instilled within the confines of the fascial sheath.

After tumescent fluid is instilled within the fascial sheath, the ablation catheter is positioned in the proximal saphenous vein. Again, this step requires the operator to be facile with the performance and interpretation of ultrasound images of the SFJ. Ideally, the tip of the ablation catheter, which represents the most proximal aspect of the ablation, should be positioned $2 \mathrm{~cm}$ distal to the SFJ. If the catheter is closer than this, there is a risk of thrombus propagating into the deep system, and thus becoming a deep vein thrombus or direct thermal injury to the CFV. If the catheter tip is further than $2 \mathrm{~cm}$ from the SFJ, there is a risk that the ablation will be inadequate. These images are best obtained in the transverse plane on gray scale imaging. Finally, color-Doppler evaluation is performed at the termination of the procedure to confirm that the GSV was successfully ablated and that there is no deep vein thrombus.

\section{Complications and postoperative surveillance}

Some of the complications associated with endovenous vein ablation include superficial thrombophlebitis, cutaneous nerve injury, and deep venous thrombosis. Rarely, development of arteriovenous fistula after treatment of the perforator has also been reported. ${ }^{26}$ Superficial thrombophlebitis is expected and treated conservatively; however, with retrograde extension it can cause pain and inflammation in remote regions. Sensory nerve injury is also reported following ablation of the GSV and is usually transient. Small saphenous ablation may be associated with sural nerve paresthesia. Injury to the superficial peroneal nerve, although rare, has been reported.

DVT is the most feared complication associated with catheter-based saphenous vein ablation. All patients have an on-table color-Doppler examination at the time of procedure 
and a repeat examination 72 hours after the procedure in our center for vascular diagnostics. DVT after open varicose vein surgery has been attributed to the procedure. After endovenous ablation, the implications of thrombus extending up to or into the CFV are not the same as for a DVT. Thus, we classify thrombus extending to the deep system after endovenous ablation as endovenous heat-induced thrombosis (EHIT). It is estimated that the incidence of thrombus extending into a deep vein after superficial venous thermoablation is $<1 \%$. ${ }^{27,28}$ Most venous thrombosis following treatment appear to be related to extension of clot from thrombus induced in the ablated vein. Direct thermal injury to the deep veins can also occur due to catheter malposition. Risk of thrombus extension into the femoral vein is associated with a history of DVT and GSV diameter $>8 \mathrm{~mm}$ at the level of the SFJ. ${ }^{29}$ Patients identified to have a DVT are treated accordingly. Patients with EHIT are further subclassified. EHIT is divided into six discrete categories: Level I where the thrombus is below superficial epigastric vein; level II: thrombus up to the origin of the superficial epigastric vein; level III: thrombus up to but not into the CFV; level IV: thrombus bulges into the $\mathrm{CFV}$; level V: thrombus extending into the CFV; and level VI: thrombus is occlusive of the CFV. In our practice, we anticoagulate patients with Level IV, V, and VI EHIT. Patients with EHIT have a repeat duplex examination after 14 days of anticoagulation. Anticoagulation is discontinued if there is thrombus resolution and is continued for 3-6 months if there is no thrombus resolution.

\section{Conclusion}

The developments in catheter-based venous treatment has largely replaced the ligation and stripping open surgery. Ultrasound is an essential component for the evaluation and management of GSV reflux. It is important for the surgeon to be familiar with both the execution and interpretation of duplex ultrasonography of the lower extremity.

\section{Disclosure}

The authors report no conflicts of interest in this work.

\section{References}

1. Beebe-Dimmer JL, Pfeifer JR, Engle JS, Schottenfeld D. The epidemiology of chronic venous insufficiency and varicose veins. Ann Epidemiol. 2005;15(3):175-184.

2. Smith JJ, Garratt AM, Guest M, Greenhalgh RM, Davies AH. Evaluating and improving health-related quality of life in patients with varicose veins. J Vasc Surg. 1999;30(4):710-719.

3. Korn P, Patel ST, Heller JA, et al. Why insurers should reimburse for compression stockings in patients with chronic venous stasis. $J$ Vasc Surg. 2002;35(5):950-957.
4. Gloviczki P, Comerota AJ, Dalsing MC, et al; Society for Vascular Surgery, American Venous Forum. The care of patients with varicose veins and associated chronic venous diseases: clinical practice guidelines of the Society for Vascular Surgery and the American Venous Forum. J Vasc Surg. 2011;53(5 Suppl):2S-48S.

5. Caggiati A, Bergan JJ, Gloviczki P, et al; International Interdisciplinary Consensus Committee on Venous Anatomical Terminology. Nomenclature of the veins of the lower limb: extensions, refinements, and clinical application. J Vasc Surg. 2005;41(4):719-724.

6. van Neer PA, Veraart JC, Neumann HA. Venae perforantes: a clinical review. Dermatol Surg. 2003;29(9):931-942. Discussion 42.

7. Zierler BK. Ultrasonography and diagnosis of venous thromboembolism. Circulation. 2004;109(12 Suppl 1):I9-I14.

8. Georgiev M, Myers KA, Belcaro G. The thigh extension of the lesser saphenous vein: from Giacomini's observations to ultrasound scan imaging. J Vasc Surg. 2003;37(3):558-563.

9. Delis KT, Knaggs AL, Khodabakhsh P. Prevalence, anatomic patterns, valvular competence, and clinical significance of the Giacomini vein. J Vasc Surg. 2004;40(6):1174-1183.

10. Meissner MH, Moneta G, Burnand K, et al. The hemodynamics and diagnosis of venous disease. J Vasc Surg. 2007;46(Suppl S):4S-24S.

11. Bonfield M, Cramp F, Robinson T. Effect of patient positioning on the duration of venous reflux in duplex ultrasound for venous insufficiency. Ultrasound. 2012;20:92.

12. Lurie F, Comerota A, Eklof B, et al. Multicenter assessment of venous reflux by duplex ultrasound. J Vasc Surg. 2012;55(2):437-445.

13. Belcaro G, Cesarone MR, Di Renzo A, et al. Foam-sclerotherapy, surgery, sclerotherapy, and combined treatment for varicose veins: a 10-year, prospective, randomized, controlled, trial (VEDICO trial). Angiology. 2003;54:307.

14. Belcaro G, Nicolaides AN, Ricci A, et al. Endovascular sclerotherapy, surgery, and surgery plus sclerotherapy in superficial venous incompetence: a randomized, 10-year follow-up trial - final results. Angiology. 2000;51:529.

15. Lurie F, Creton D, Eklof B, et al. Prospective randomised study of endovenous radiofrequency obliteration (closure) versus ligation and vein stripping (EVOLVeS): two-year follow-up. Eur J Vasc Endovasc Surg. 2005;29:67.

16. Rasmussen LH, Bjoern L, Lawaetz M, Blemings A, Lawaetz B, Eklof B. Randomized trial comparing endovenous laser ablation of the great saphenous vein with high ligation and stripping in patients with varicose veins: short-term results. J Vasc Surg. 2007;46:308.

17. Kalteis M, Berger I, Messie-Werndl S, et al. High ligation combined with stripping and endovenous laser ablation of the great saphenous vein: early results of a randomized controlled study. J Vasc Surg. 2008; $47: 822$.

18. Almeida JI, Kaufman J, Göckeritz O, et al. Radiofrequency endovenous ClosureFAST versus laser ablation for the treatment of great saphenous reflux: a multicenter, single-blinded, randomized study (RECOVERY study). J Vasc Interv Radiol. 2009;20:752.

19. Bacon JL, Dinneen AJ, Marsh P, Holdstock JM, Price BA, Whiteley MS. Five-year results of incompetent perforator vein closure using TRansLuminal Occlusion of Perforator. Phlebology. 2009;24:74.

20. Masuda EM, Kessler DM, Lurie F, Puggioni A, Kistner RL, Eklof B. The effect of ultrasound-guided sclerotherapy of incompetent perforator veins on venous clinical severity and disability scores. J Vasc Surg. 2006;43:551.

21. Luebke T, Brunkwall J. Meta-analysis of subfascial endoscopic perforator vein surgery (SEPS) for chronic venous insufficiency. Phlebology. 2009;24:8.

22. Fegan WG. Continuous compression technique of injecting varicose veins. Lancet. 1963;2:109.

23. Stacey MC, Burnand KG, Layer GT, Pattison M. Calf pump function in patients with healed venous ulcers is not improved by surgery to the communicating veins or by elastic stockings. Br J Surg. 1988;75(5): 436-439. 
24. Gloviczki P, Comerota AJ, Dalsing MC, et al; Society for Vascular Surgery, American Venous Forum. The care of patients with varicose veins and associated chronic venous diseases: clinical practice guidelines of the Society for Vascular Surgery and the American Venous Forum. J Vasc Surg. 2011;53:2S.

25. O'Donnell TF Jr, Passman MA. Clinical practice guidelines of the Society for Vascular Surgery (SVS) and the American Venous Forum (AVF) - management of venous leg ulcers. Introduction. J Vasc Surg. 2014;60:1S.

26. Bacon JL, Dinneen AJ, Marsh P, Holdstock JM, Price BA, Whiteley MS. Five-year results of incompetent perforator vein closure using TRansLuminal Occlusion of Perforator. Phlebology. 2009;24:74.
27. Mozes G, Kalra M, Carmo M, et al. Extension of saphenous thrombus into the femoral vein: a potential complication of new endovenous ablation techniques. J Vasc Surg. 2005;41:130.

28. Hingorani AP, Ascher E, Markevich N, et al. Deep venous thrombosis after radiofrequency ablation of greater saphenous vein: a word of caution. J Vasc Surg. 2004;40:500.

29. Lawrence PF, Chandra A, Wu M, et al. Classification of proximal endovenous closure levels and treatment algorithm. J Vasc Surg. 2010;52:388.

\section{Publish your work in this journal}

Journal of Vascular Diagnostics is an international, peer-reviewed journal of diagnostics, focusing on non invasive vascular investigation methods involved in the evaluation of vascular diseases. The journal is committed to the rapid publication in the fields of vascular diseases. Original research, review, case reports, expert opinion and commentaries

\section{Dovepress}

are all considered for publication. The manuscript management system is completely online and includes a very quick and fair peer-review system, which is all easy to use. Visit http://www.dovepress.com/testimonials.php to read real quotes from published authors.

Submit your manuscript here: http://www.dovepress.com/journal-of-vascular-diagnostics-journal 